\title{
Throughput improvement in partially crowded indoor areas
}

\author{
Deusdedith Ndaboine ${ }^{1}$ and Herald N. Kundaeli ${ }^{2}$ \\ ${ }^{I}$ Department of IT \& Services, MIC Tanzania, PO Box 2929, Dar es Salaam, TANZANIA \\ ${ }^{2}$ Department of Electronics and Telecommunications Engineering, COICT, PO Box 33335, Dar es Salaam, TANZANIA \\ Corresponding Author: kundaelh@yahoo.com
}

\begin{abstract}
In recent years there has been a dramatic increase in the number of people using advanced mobile devices, leading to a jump in the number of mobile broadband subscriptions and network traffic. In most instances such users tend to operate in indoor areas which then become crowded. The resulting high density of demand for connection to the network and the attendant interference among the user devices tend to overload the network and degrade its performance. This paper reports on the deployment of small cells in crowded indoor areas, as a solution to provide broadband services to the users. The results show that improved performance is achieved when small cells are deployed to offload traffic from the macro cells.
\end{abstract}

Keywords: Indoor broadband, LTE, Small cells, Throughput.

DOI: http://dx.doi.org/10.4314/ijest.v11i4.1

\section{Introduction}

The telecommunication industry has surpassed other industries in the world today in terms of size and growth rate. This is attributed to the ever increasing demand by the society for more services, delivered anywhere needed, and delivered as fast as possible. The result is a continuously evolving global mobile communication infrastructure, with increased number of subscribers amounting to one billion in 2002, and rising to five billion in the 2010's (Holma and Toskala, 2011). The requirements for service delivery during mobility has led to a higher increase in the mobile phone sector compared to the fixed phone sector. In Tanzania for example, the statistics in Figure 1 (Appendix) shows an increase of $31.5 \%$ in the mobile phone subscriptions at the expense of fixed phone subscriptions from 2013 to 2017 (TCRA, 2018).

Recent studies have shown that while some people access mobile internet in open areas, an increasing number are accessing the mobile internet in indoor areas like markets, schools and offices (Taylor, 2011). As a result, the indoor areas become semipermanently crowded, $80 \%$ most of the time. The features of the buildings in the indoor areas, however, affect the penetration of radio signals at high frequencies, leading to fading. Such features include building size, shape, structure, room layout and type of construction materials. In particular, the variations in the features of the buildings cause the signals to fade faster at the high frequencies used in broadband communication. As a result, users operating in indoor areas have poor communication with the macro base transceiver stations (BTS) positioned outdoors.

The increase in the use of mobile internet provided the motivation to develop new communication technologies by the 3GPP (Third Generation Partnership Program). Such technologies include the Long-Term Evolution (LTE) and the Long-Term Advanced (LTE-A). These technologies have overtaken GSM/EDGE in catering for the mobile communication subscribers, who are expected to rise to 2.6 billion globally in 2019, and representing the major subscribers (Ericsson, 2013).

The primary objective of this research was to overcome the limitations faced by mobile internet users in semi-permanently occupied indoor areas, and thus enable them to benefit from the new LTE and LTE-A technologies. It was in particular to improve the throughput in these areas by setting up femtocells through a number of steps. First, establishing the throughput, including its variation arising from the indoor path loss which then represents a semi-permanently crowded indoor area. Second, establishing how the throughput is affected by deploying small cells randomly in the chosen indoor areas. Third, establishing the performance 
in throughput when LTE-A is used for backhauling to small cells in the chosen indoor area. Finally, to evaluate the performance in throughput for the LTE users attached to the small cells in the indoor areas. Since ray tracing is the dominant method of analyzing wave propagation in small cells environments, we start with a short introduction to it.

\section{Ray Tracing Propagation Model}

The loss propagation of radio waves in rural, urban and semi-urban environments, and in different materials have being widely studied and several models have been developed (Rappaport, 2002). Similar studies in indoor environments have become important as wireless cellular communication has gradually adopted micro, pico and femto cells over macro cells. Unfortunately, the large scale theoretical and empirical models employed in outdoor environments fail to yield good results in indoor environments and wave propagation modeling using ray tracing has become the main method of investigation. A typical radio propagation scenario that calls for ray tracing techniques is shown in Figure 2.

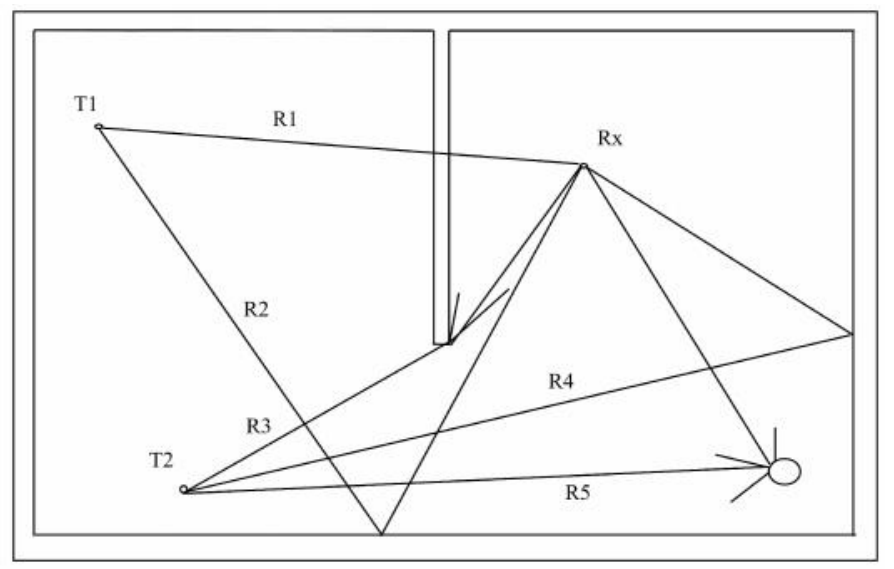

Figure 2: Sample rays for two transmitters and one receiver in a room.

It shows rays R1 to R5 from transmitters $\mathrm{T} 1$ and $\mathrm{T} 2$ that suffer reflection, diffraction, scattering and wall losses before reaching receiver Rx. Detailed treatment of methods of ray tracing can be found in (Yun and Iskanderr, 2015; Hussain, 2017; He et al., 2018), while the applications of ray tracing in the Winprop software can be found in (AWE Communications, 2012; Jakobus et. al. 2018; Fagiani et. al., 2018; Kelmendi, 2018).

\section{Methodology}

The research methodology adopted noted that the purpose of the study was to improve the transmission throughput in indoor areas that are semi-permanently crowded using the commercial WinProp (AWE Communications, 2012) wave propagation modeling tool. The study area chosen to perform the network simulation was the Teaching block of the College of ICT (CoICT) of the University of Dar es Salaam, Tanzania, whose 3-D image is shown in Figure 3.

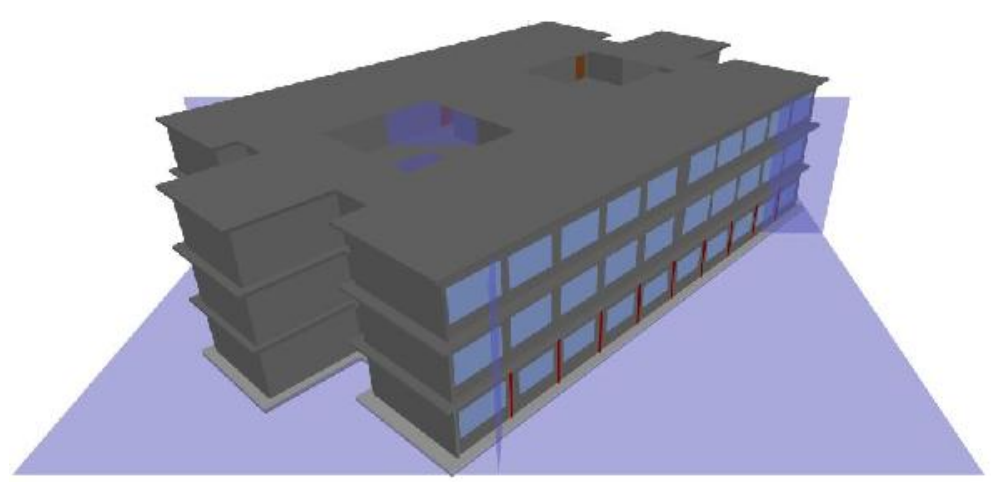

Figure 3: The 3D architecture of the study area. 
The Teaching block was chosen for two reasons. One, it fits the characteristics of semi-permanently crowded indoor areas with varying occupancy by students who require high throughput internet connectivity for accessing online study material. Two, the 2D floor images and measurements for generating the 3D image to be used in propagation modelling were also readily available. In order to derive the transmission limitations of the building, the properties of the materials were measured and recorded and then used in the simulation for propagation modelling using the WinProp software package. The obtained loss data was then used in statistical analysis to compute the throughput using the modified Shannon's formulation for LTE capacity equation in the Matlab software.

A heterogeneous network deployment scenario for the study is shown in Figure 4. The users in the same service site were deployed in small cells and LTE macro cells. In total there were 30 macro cells and 8 small cells. Basing on the analysis software tool (AWE Communications, 2014), two scenarios were employed. In the baseline scenario only 30 Macro cells were deployed, while 8 small cells were deployed per floor on top of the 30 Macro cells in the small cells scenario.

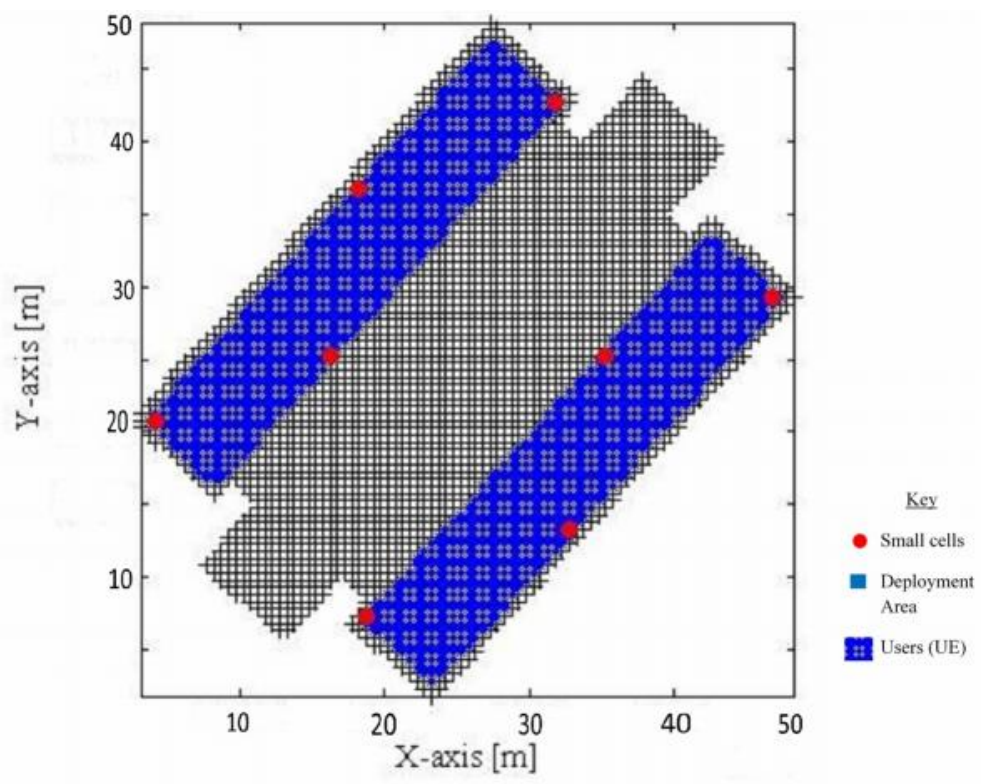

Figure 4: CoICT indoor deployment scenario.

Four operating frequencies were used for the simulation, $800 \mathrm{MHz}, 2000 \mathrm{MHz}, 2600 \mathrm{MHz}$ and $5000 \mathrm{MHz}$ using a Kathrein antenna. The four frequencies were taken as adequate for the study because they span the range of frequencies in which LTE can be used. Three categories of data were collected, the signals reaching the indoor users through the small cells, the signals reaching the small cells through the backhauling links, and the throughputs of the UEs for the different categories of backhaul links and sizes of the carriers.

\section{Results and Discussions}

The results of the study are given in Figures 5 to 11 and Tables 1 to 3 . The throughput performance of the user equipment (UE) in macro-cells is given in Figure 5 while in comparison the UE performance operating in small cells is given in Figure 6 . The comparison was done at $800 \mathrm{MHz}$ as the operating frequency with the carrier bandwidth, the backhauling frequencies and the number of UEs in the chosen indoor area remaining unchanged. As seen from both Figures 5 and 6, when only $10 \%$ of the UEs were operational the small cells configuration supported a throughput of $1 \mathrm{Mbps}$ whereas the macro cells configuration supported only 0.4 Mbps. This indicates that the small cells configuration is bound to support higher throughput than the macro cells configuration. Using the results in Table 1, the throughput attained by UEs in the small cells with $10 \%$ CDF of the UEs being idle exceeds the one attained by the macro cells by a factor of 13. Likewise, when $90 \%$ CDF of UEs are idle, the throughput attained by UEs in small cells exceeds the one attained in macro cells by a factor of 22. The results reported in (Yavuz et. al., 2009) also show that the use of small cells increase the throughput of UEs.

The variation in the performance of the UEs with frequency is presented in Table 2 where the backhaul link was assumed to be ideal. As can be seen, the throughput increases with operating frequency, being higher at 5,000 MHz compared to $800 \mathrm{MHz}$. For example, in operations having 50\% of UE idle the throughput is $7.497 \mathrm{Mbps}$ at 5,000 MHz, while the throughput at $800 \mathrm{MHz}$ is half of that. The presented results also show that when the percentile of idle UEs increases, the throughput also increases. For example at 5,000 MHz, the throughput is $19.008 \mathrm{Mbps}$ at $90 \%$ idle UEs. This is more than twice the throughput at 50\% idle UEs, 
and it also exceeds the throughput at $10 \%$ idle UEs by a factor of 8 . These results also show that better performance can be obtained at higher frequencies.

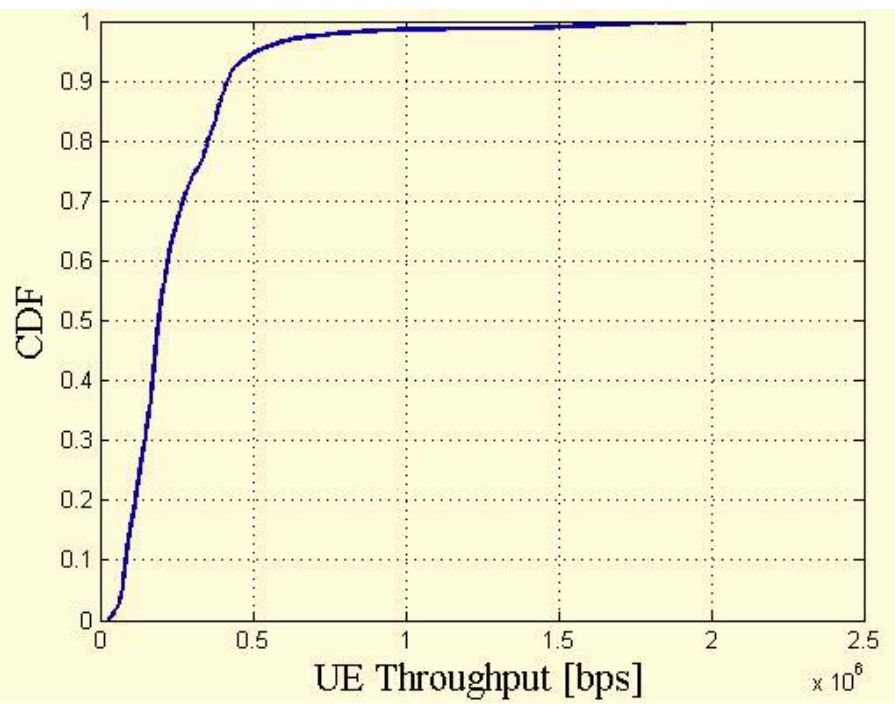

Figure 5: Throughput of UE operating at $800 \mathrm{MHz}$ in macro cells.

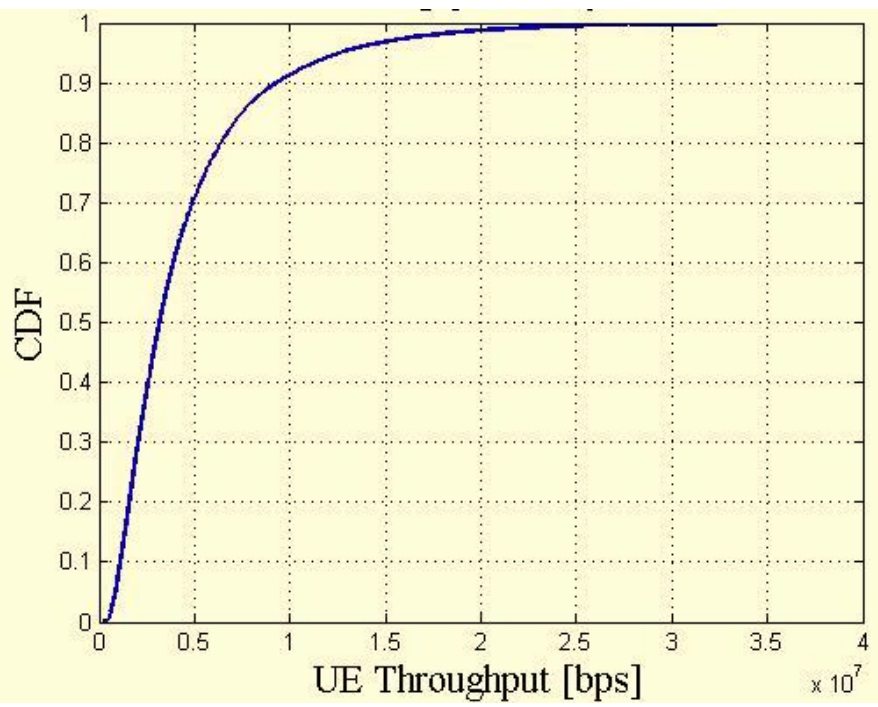

Figure 6: Throughput of UE operating at $800 \mathrm{MHz}$ in small cells.

Table 1: Throughput of UEs operating at $800 \mathrm{MHz}$ in macro cells and small cells.

\begin{tabular}{|l|l|l|l|}
\hline Cell Type & \multicolumn{3}{|c|}{ Throughput (Mbps) } \\
\hline & $\mathbf{1 0 \%}$ (Idle UEs) & $\mathbf{5 0 \%}$ (Ideal UEs) & $\mathbf{9 0 \%}$ (Idle UEs) \\
\hline Macro Cell & 0.08490 & 0.19230 & 0.41754 \\
\hline Small Cell & 1.1274 & 3.1811 & 9.2861 \\
\hline
\end{tabular}

Table 2: Throughput of UE operating in small cells at the four frequencies

\begin{tabular}{|l|c|c|c|c|}
\hline Idle UEs & \multicolumn{4}{|c|}{ Throughput (Mbps) } \\
\hline & $\mathbf{8 0 0} \mathbf{~ M H z}$ & $\mathbf{2 , 0 0 0} \mathbf{~ M H z}$ & $\mathbf{2 , 6 0 0} \mathbf{~ M H z}$ & $\mathbf{5 , 0 0 0} \mathbf{~ M H z}$ \\
\hline $\mathbf{1 0 \%}$ & 1.1274 & 1.702 & 1.702 & 2.242 \\
\hline $\mathbf{5 0 \%}$ & 3.1811 & 5.102 & 5.102 & 7.497 \\
\hline $\mathbf{9 0 \%}$ & 9.2861 & 14.148 & 14.148 & 19.008 \\
\hline
\end{tabular}


The variation in the quality of the signal received by the UEs with frequency is presented in Figure 7.

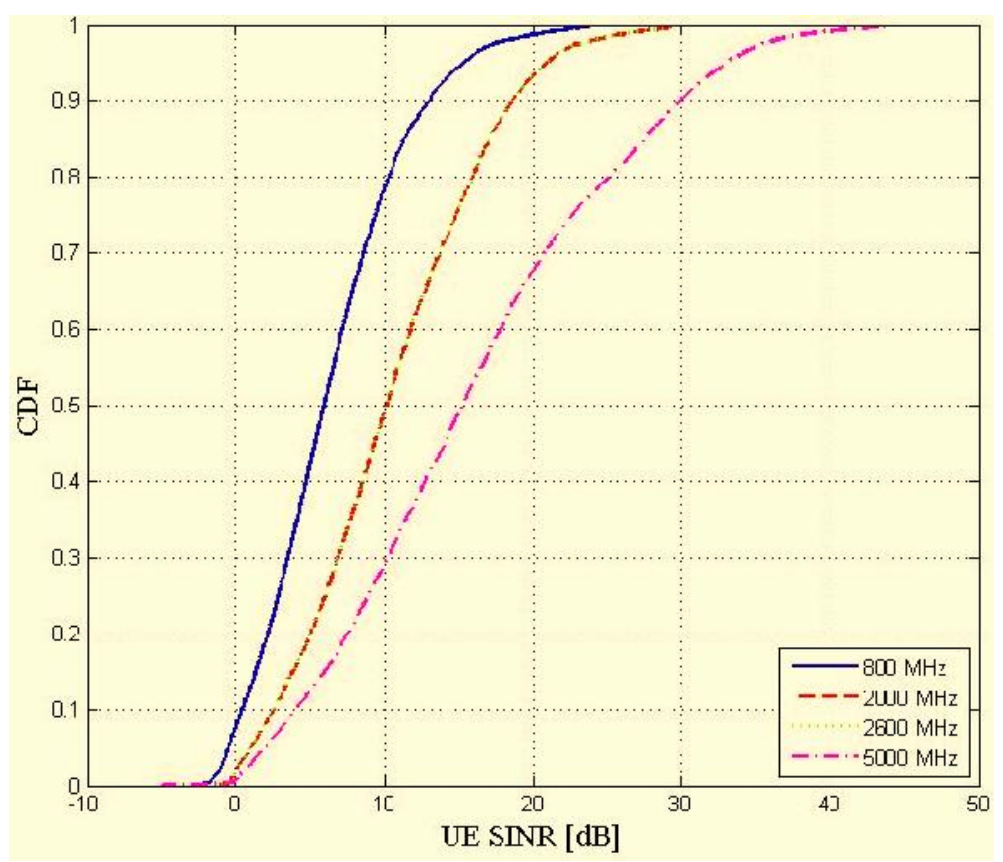

Figure 7: The SNIR of UEs at the

four frequencies

In this case it was assumed that the backhaul link remained ideal. As seen, the Signal to Interference Noise Ratio (SINR) attained by the UE at $800 \mathrm{MHz}$ was found to be significantly lower compared to the SINR obtained at 5,000 MHz. For example, at 50\% the SINR for 5,000 MHz was found to be 2 times the SINR at $800 \mathrm{MHz}$. However, the SINR obtained at 2,000 MHz was almost the same as the SINR obtained at $2,600 \mathrm{MHz}$, and therefore the study concentrated more on $2600 \mathrm{MHz}$ since the results at this frequency also apply to 2,000 MHz. Another quality of the received signal is Spectral Efficiency (SE), which is presented in Figure 8 at the four frequencies. The results show that the SE at higher frequencies is slightly higher compared to the SE at lower frequencies. The implication here is that at higher frequencies the UEs utilize the bandwidth more efficiently while at lower frequencies the utilization is poor.

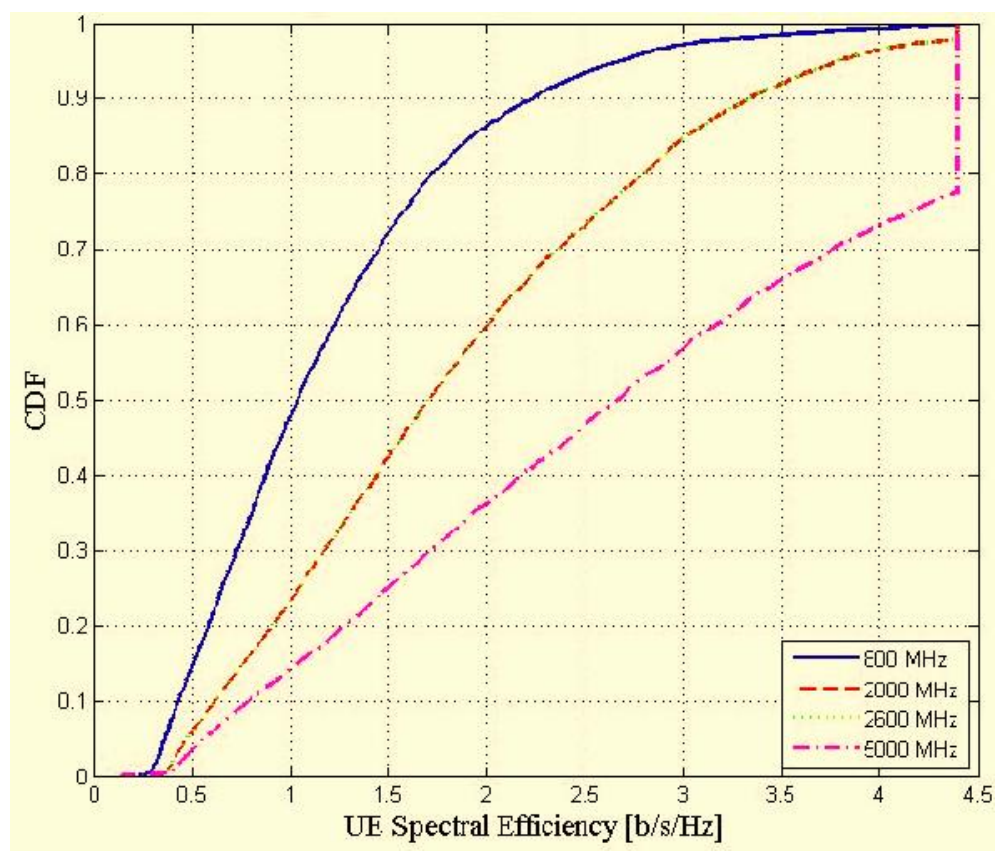

Figure 8: The spectral efficiency of UE at the four frequencies 
In this study, 8 UEs were deployed in the indoor area. The target in this case was to investigate how the throughput achieved by the UEs in the small cells varied with the macro cells backhaul frequency. As shown in Figure 9, there is throughput difference between the frequencies at the 10 percentile, which increases from the 50 percentile upwards. This difference reaches about 14 Mbps at the 90 percentile, indicating that UEs in small cells attain better performance at high frequencies.

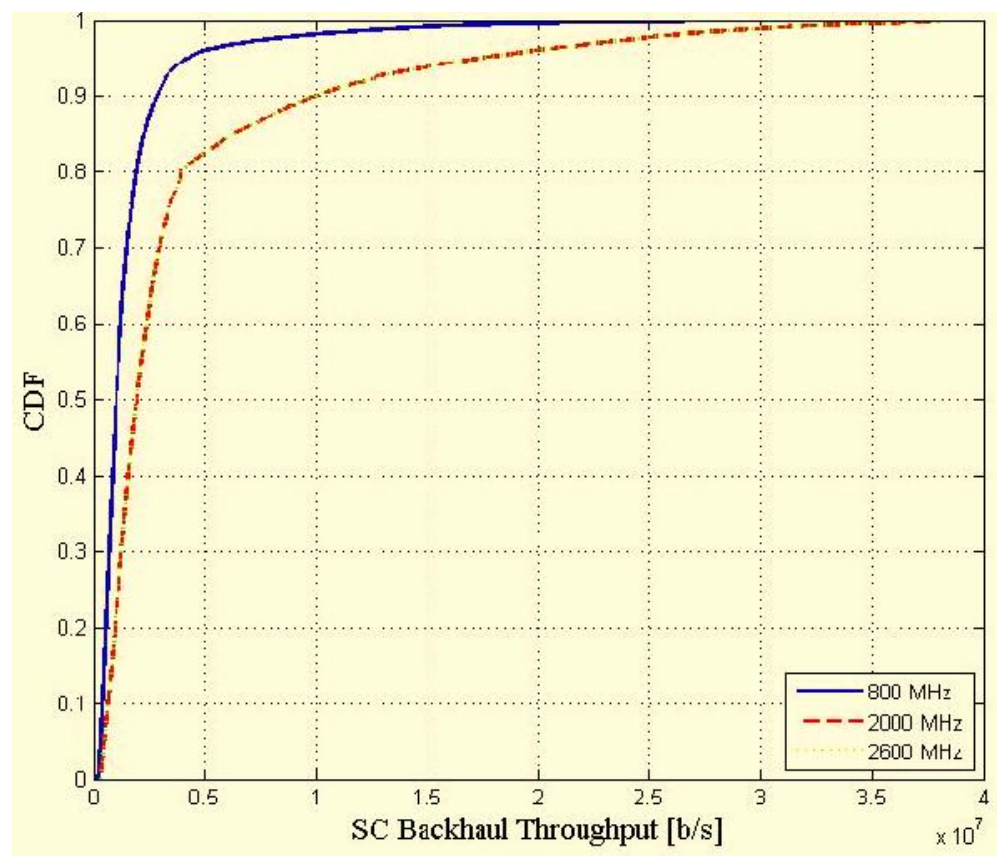

Figure 9: Throughput in SC backhaul at different frequencies

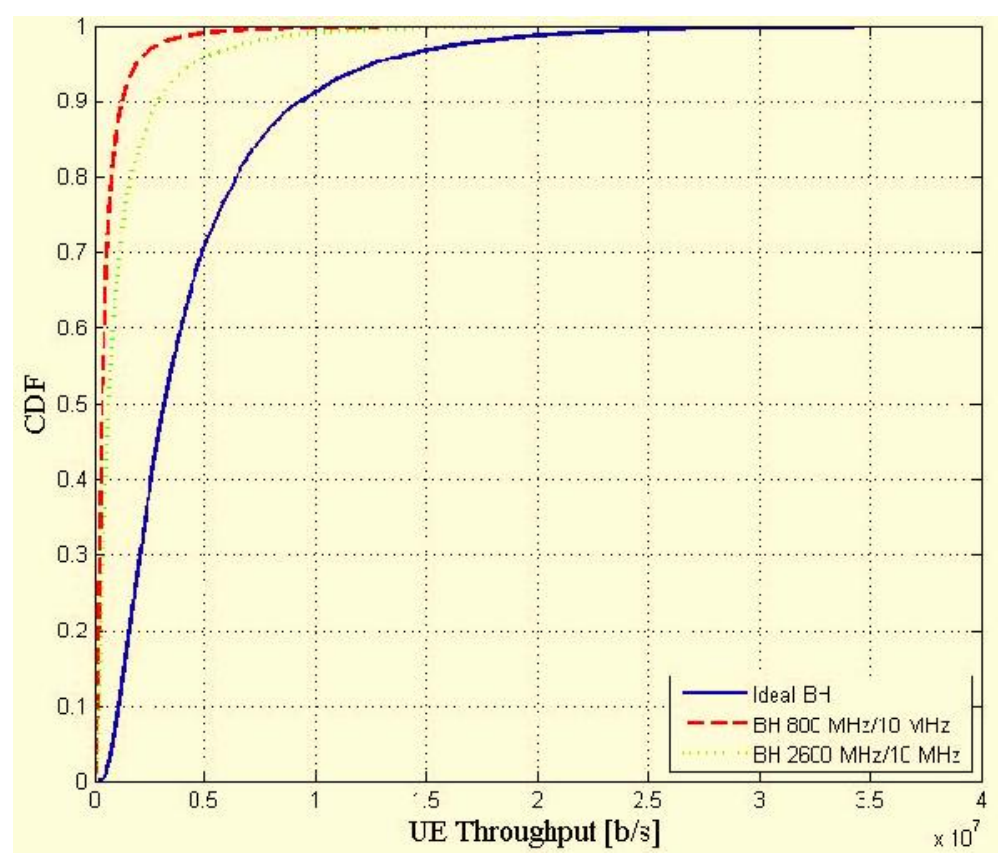

Figure 10: Throughput of UEs at $800 \mathrm{MHz}$, different backhaul links and $10 \mathrm{MHz}$ carrier bandwidth.

The throughput of the UEs was found to depend on their operating frequency, the employed backhaul frequency, and the allocated bandwidth of the carriers. The variation of the throughput with the backhaul frequency of $800 \mathrm{MHz}$ and a carrier bandwidth of $10 \mathrm{MHz}$ is presented in Figure 10. The results show insignificant difference in throughput between the backhaul frequencies of $800 \mathrm{MHz}$ and 2,600 MHz when the fraction of idle UEs ranges from $10 \%$ to $50 \%$. The variation in the throughput when the operating frequency was raised to $5,000 \mathrm{MHz}$ is seen in Figure 11. As seen, there is improved performance at 5,000 
$\mathrm{MHz}$, but there is also much difference with the backhaul frequencies. The observation here is that the lower frequencies are more prone to interference resulting in poor throughput, and this has been deduced in (Intracom, 2013).

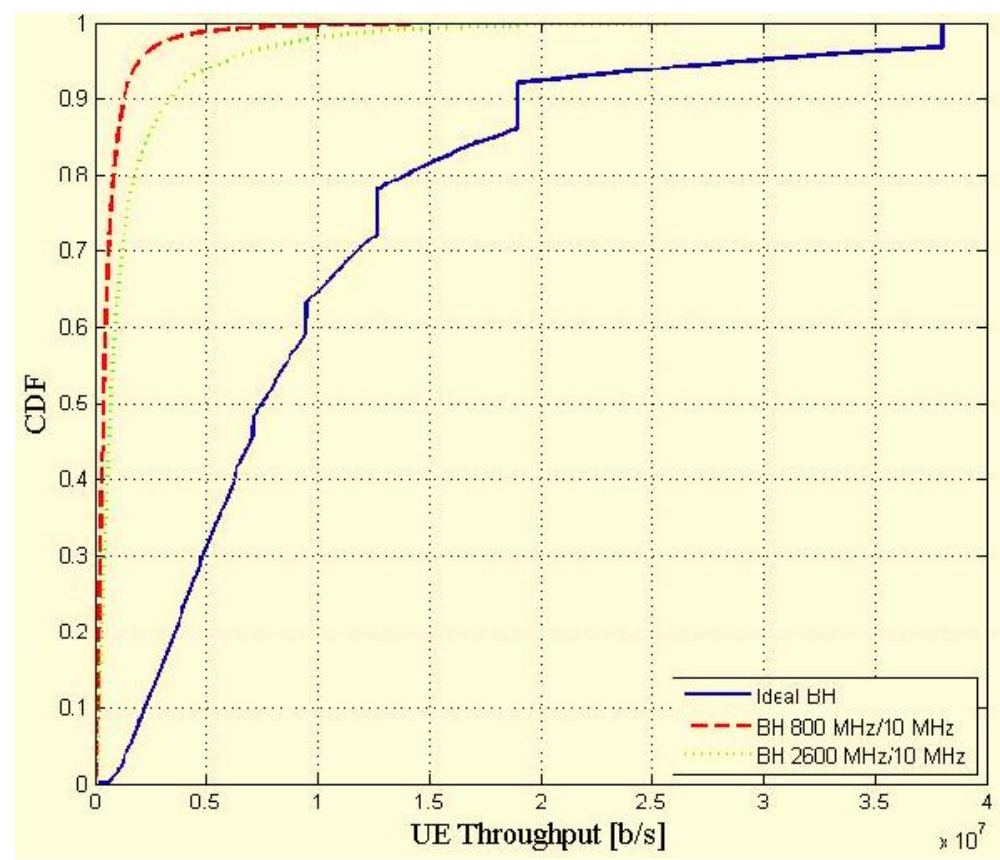

Figure 11: Throughput of UE at $5000 \mathrm{MHz}$, different backhaul links and $10 \mathrm{MHz}$ carrier bandwidth.

More comparative results are presented in Table 3. These results show that generally higher backhaul frequencies support higher throughput, and higher bandwidths support higher throughputs.

Table 3: Throughput at different operating and backhaul frequencies, and carrier size

\begin{tabular}{|c|c|c|c|}
\hline & \multicolumn{3}{|c|}{ Throughput (Mbps) } \\
\hline $\begin{array}{l}\text { Frequencies: Operation, } \\
\text { backhauling, carrier size }\end{array}$ & $10 \%$ (Idle UEs) & $50 \%$ (Idle UEs) & $90 \%$ (Idle UEs) \\
\hline $800 \mathrm{MHz}, 800 \mathrm{MHz}, 10 \mathrm{MHz}$ & 0.1239 & 0.3757 & 1.2790 \\
\hline $800 \mathrm{MHz}, 2,600 \mathrm{MHz}, 10 \mathrm{MHz}$ & 0.2002 & 0.6836 & 2.8692 \\
\hline $5,000 \mathrm{MHz}, 800 \mathrm{MHz}, 10 \mathrm{MHz}$ & 0.1234 & 0.3748 & 1.3020 \\
\hline $5,000 \mathrm{MHz}, 2,600 \mathrm{MHz}, 10 \mathrm{MHz}$ & 0.2007 & 0.6909 & 3.3446 \\
\hline $800 \mathrm{MHz}, 800 \mathrm{MHz}, 20 \mathrm{MHz}$ & 0.2553 & 0.7713 & 2.5476 \\
\hline $800 \mathrm{MHz}, 2,600 \mathrm{MHz}, 20 \mathrm{MHz}$ & 0.3983 & 1.2794 & 4.4575 \\
\hline $5,000 \mathrm{MHz}, 800 \mathrm{MHz}, 20 \mathrm{MHz}$ & 0.2536 & 0.7773 & 2.6480 \\
\hline $5,000 \mathrm{MHz}, 2,600 \mathrm{MHz}, 20 \mathrm{MHz}$ & 0.4012 & 1.3558 & 5.7384 \\
\hline
\end{tabular}

In employing the small cells solution for improved performance in indoor environments, SINR, spectral efficiency, backhaul frequencies and bandwidths were considered. First, small cell networks are seen as the solution to support the increase in broadband wireless usage in indoor environments (Intracom Telecom, 2013; Wang et al, 2014). The higher throughput illustrated in this study advocate the establishment of small cells in crowded indoor environments. In this study the effects of SINR and spectral efficiency on the quality of the communication have been demonstrated. These have to be dealt with in future communications as small cells become more adopted and the density of users in them increases (Yang et. al., 2016). An even more important factor is the frequency resource. This study has revealed that for increased throughput, higher frequencies and larger bandwidths are preferable. However, the operating frequencies and bandwidths become more important as small cells networks become congested, and innovative solutions for their utilizations need to be developed (Hwang, et. al., 2013).

In this study attempts were not made to mitigate interference in the macro-cell small cell environment. In the report of Yavuz et. al. (2009) interference was managed by programming the transmitters to choose frequencies where interference was lower, as well as to limit their transmission power in order to avoid excessive interference. In both reports, however, the utilization of radio resources was considered with the CDF representing the percentage of users who were idle. Thus, a CDF of $10 \%$ idle UEs translates to $90 \%$ of UEs being active and therefore implying a high utilization of resources. The results therefore provide 
performance in throughput that is tagged to the utilization of resources. In addition, the UE's were randomly deployed as seen in Figure 4, and they equally shared the resources as they were both indoor users served by the small cells. Moreover, unlike the study of Yavus et. al. (2009), no efforts were made in this study to use higher energy values in order to overcome interference or the losses in the building wall materials. The results in this report indicate that the use of small cells increases throughput, as seen by comparing Figures 5 and 6. Therefore, despite the interference, the deployment of small cells improves QoS since the UEs will not suffer much from signal penetration losses through the buildings. The increase of more UEs in a macro cell makes the network more complex because the macro cell transmitters have to handle many more connection requests. On the other hand, the deployment of small cells offloads a good number of the macro cell connection requests to the indoor small cells, thus reducing the demands on the outdoor macro cells, and thus reducing the network complexity. Therefore, considering all the factors associated with the introduction of small cells in macro cell environments, interference remains as the factor that has to be tackled.

\section{Conclusions}

The study aimed at improving the transmission performance in partially crowded areas has been undertaken. The focus was on the use of small cells and the different technologies that support the communicating devices, capitalizing on LTE and LTE-A as future technologies, and the backhauling that support the small cells. The obtained results also indicate how a suitable radio wave prediction tool can be employed in order to alleviate the burden of computational complexity and resources associated with ray tracing methods. While the obtained results support the use of small cells for higher throughput and quality of user experience, factors like configurable network architecture, spectral efficiency, operating frequencies, and bandwidth need to be taken into consideration to meet the explosion of data traffic usage. More research is therefore needed aimed at developing more innovative solutions to maximize resource usage while maintaining quality of communication in the small cells technology.

\section{Appendix}

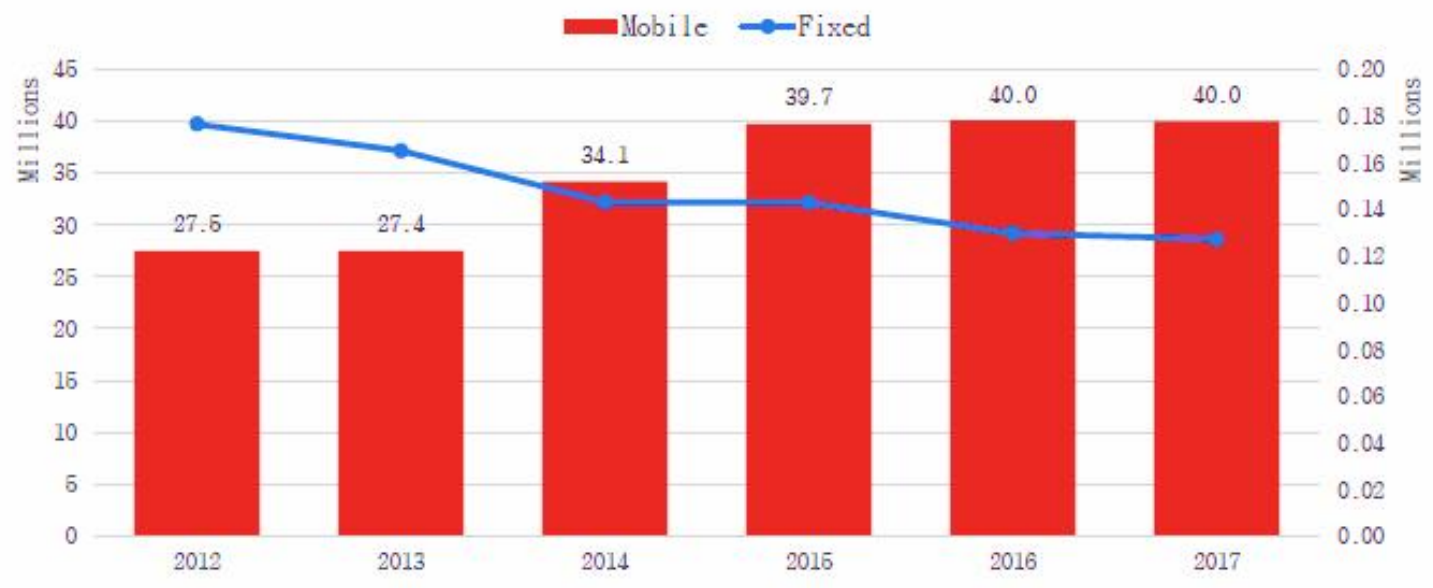

Figure 1: Trends of mobile and fixed-line telephone subscriptions in Tanzania (TCRA, 2018).

\section{Acknowledgements}

The authors acknowledge the College of Information and Communication Technologies (COICT), University of Dar es Salaam, who supported this research. Acknowledgements are also due to the various reviewers whose comments helped to improve the report.

\section{References}

AWE Communications. 2012. Indoor Propagation. Available at: http://www.awe-communications.com/Propagation/Indoor/ (Accessed: 13 February 2014).

AWE Communications. 2014. Indoor Scenarios. Available at: http://www.awe-communications.com/Propagation/Indoor/ (Accessed: 1 April 2014).

Ericson. 2013. Ericsson Mobility Report: On the Pulse of the Networked Society .Available at: https://www.ericsson.com/assets/local/mobility-report/documents/2016/ (Accessed: 15 February 2014).

Fagiani, A., Vogel, M. and Cerqueira, A. 2018. Material Characterization and Propagation Analysis of mm-Waves Indoor Networks. Journal of Microwaves, Optoelectronics and Electromagnetic Applications. Vol. 17, No. 4, pp.628-637. 
He, D., Ai, B., Guan, K., Wang, L., Zhong, Z. and Kürner, T. 2018. The Design and Applications of High-Performance RayTracing Simulation Platform for 5G and Beyond Wireless Communications: A Tutorial. IEEE Communications Surveys \& Tutorials, Vol. 21, No. 1, pp.10 - 27.

Holma, H. and Toskala, A. 2011. LTE for UMTS: Evolution to LTE-Advanced. $\left(2^{\text {nd }}\right.$ Ed). Finland: John Wiley \& Sons Ltd. Hussain, S. 2017. Efficient Ray-Tracing Algorithms for Radio Wave Propagation in Urban Environments. PhD Thesis, Dublin City University.

Hwang, I., Song, B. and Soliman, S. S. (2013). A holistic view on hyper-dense heterogeneous and small cell networks. IEEE Communications Magazine, Vol. 51, No. 6, pp.20-27.

Intracom Telecom. 2013. Technology Synergies for Small-Cell Backhaul in 4G Networks. Athens. Available at: http://www.intracom-telecom.com/downloads/pdf/products/wireless_access/wp/ (Accessed: 11 May 2014).

Jakobus, U., Aguilar, A. G., Woelfle, G., van Tonder, J., Bingle, M., Longtin, K. and Vogel, M. 2018. Recent Advances of FEKO and WinProp. 2018 IEEE International Symposium on Antennas and Propagation \& USNC/URSI National Radio Science Meeting, 8-13 July 2018, Boston, MA, USA. DOI: 10.1109/APUSNCURSINRSM.2018.8608939.

Kelmendi, A., Jovarnik, T., Ozimek, I and Vilhar, A. 2018. Performance of ITU-R P.1546 Propagation Model. 11th IEEE-IET Interm. Symposium on Communication Systems, Networks and Digital Signal Processing. 18-20, July 2018, Budapest, Hungary, pp.141-150.

Rappaport, T. S. 2002. Wireless Communications: Principles and Practice. New York: Prentice-Hall.

TCRA. 2018. Quarterly Telecommunications Statistics. Dar es Salaam, Tanzania.

Taylor, S. 2011. A New Chapter for Mobile? San Jose: Cisco Internet Business Solutions Group.

Wang, H., Zhou, X. and Reed, M. C. 2014. Coverage and Throughput Analysis with a Non-Uniform Small Cell Deployment. IEEE Transactions on Wireless Communications, Vol. 13, No. 4, pp.2047-2059.

Yang, C., Li, J. and Guizani, M. 2016. Cooperation for spectral and energy efficiency in ultra-dense small cell networks. IEEE Transactions on Wireless Communications, Vol. 23, Vol. 1, pp.64-71.

Yavuz, M., Meshkati, F. and Nanda, S. 2009. Interference Management and Performance Analysis of UMTS/HSPA+ Femtocells. IEEE Communications Magazine, Vol. 27, No. 9, pp.102-103.

Yun, Z. and Iskanderr, M. F. 2015. Ray Tracing for Radio Propagation Modeling: Principles and Applications, IEEE Access, Vol.3, pp.1089-1100.

\section{Biographical notes}

Deusdedith Ndaboine received his BSc and MSc from the University of Dar es Salaam in 2010 and 2017 respectively. He is currently working as a Telecommunication Engineer in charge of Consumer Products Supervision at Tigo Tanzania. His interests in technology include Advanced Wireless Communication, Internet of Things, Artificial Intelligence and Robotic Process Automation.

Herald N. Kundaeli received his B. Sc., M.Sc. and PhD degrees in 1979, 1982 and 1996 respectively. He has conducted teaching and supervision of undergraduate and postgraduate students, research and consultancies in electronics and telecommunications at the University of Dar es Salaam since 1979. He is currently an Associate Professor in the Department of Electronics and Telecommunication Engineering at the University of Dar es Salaam, Tanzania. His expertise and research interests are in electronics, wireless communication and optical fibre communication; with specialities in synchronisation and error control, signal processing, and the applications of mathematical models to interdisciplinary research.

Received March 2019

Accepted May 2019

Final acceptance in revised form July 2019 